\title{
Fabrication and properties of composites of
}

\section{poly(ethylene oxide) and functionalized carbon nanotubes}

\author{
H.Z. Geng ${ }^{1}$, R. Rosen ${ }^{1}$, B. Zheng ${ }^{2}$, H. Shimoda ${ }^{1}$, L. Fleming ${ }^{1}$, J. Liu ${ }^{2}$, O. Zhou ${ }^{1,3}$
}

(1) Curriculum in Applied and Materials Sciences

University of North Carolina at Chapel Hill, Chapel Hill, NC 27599

(2) Department of Chemistry

Duke University, Durham, NC 27708

(3) Department of Physics and Astronomy

University of North Carolina at Chapel Hill, Chapel Hill, NC 27599 
Carbon nanotubes (CNTs) $[1,2]$ have dimensions similar to those of typical polymeric chains, with $1-50 \mathrm{~nm}$ in diameter and $1-10 \mu \mathrm{m}$ in length. Experimental results on individual carbon nanotubes and nanotube bundles show that they have exceptional electrical and mechanical properties with an elastic modulus of $\sim 1 \mathrm{TPa}$ and fracture strain of $5-10 \%$. [3] The thermal conductivity of the nanotubes is expected to be higher than that of the carbon fibers because of their structural perfection. As a result there are considerable interests in utilization of CNTs as fillers for thermal management and structural reinforcement. Although there have been several published studies on polymer-CNT composites [4-9], realization of the expected enhancement in properties, especially mechanical properties, has by and large not been demonstrated. This is in part because of the difficulties in materials processing arising from insolubility of the pristine CNTs.

Recent studies have shown that CNTs can be chemically functionalized such that they are soluble in selected solvents. $[10,11]$ Mickelson et al. reported that fluorinated SWNTs (F-SWNTs) were dissolved in 2-propanol at a concentration of $1 \mathrm{mg} / \mathrm{mL}$ at room temperature. [12] The nanotubes appear to be intact and are electrically insulating after fluorination. The enhanced solubility and potential of tailoring the interfacial bonding between the nanotubes and the matrix by modifying the side-groups make functionalized CNTs attractive fillers for composites. Here we report results from initial studies of composites of F-SWNTs and a semi-crystalline thermoplastic polymer, poly-(ethylene oxide) (PEO). Electron microscopy and thermal analysis showed that they have improved uniformity and nanotube dispersion compared with those by pristine SWNTs. Significant enhancement of the mechanical properties was obtained. The storage modulus (E') and yield strength increased monotonically with increasing F-SWNT loading. At 4wt\% loading, the room temperature E' is $400 \%$ of that of the control sample. Further analysis indicates strong interfacial bonding between the F-SWNTs and the PEO matrix. 
Single-walled nanotube bundles (SWNTs) [13] were synthesized by the laser ablation method under conditions described elsewhere. [14] The raw materials were purified to remove the impurity phases such as amorphous carbon and graphitic nanoparticles by reflux in $\mathrm{H}_{2} \mathrm{O}_{2}$ followed by filtration. [15] Transmission electron microscopy (TEM) and x-ray diffraction studies show that the samples contain over $90 \%$ SWNT bundles with the average bundle diameter of $30-50 \mathrm{~nm}$, tube diameter of $1.4 \mathrm{~nm}$ and bundle length of $5-10 \mu \mathrm{m}$. For the fluorination reaction, $10-15 \mathrm{mg}$ purified SWNTs were dispersed in dimethylformamide (DMF) by sonication. Filtering the solution through a Nylon filter membrane (Milipore, pore size $0.45 \mu \mathrm{m}$ ) resulted in a black film on the surface. The film was then peeled off and baked in air at $100^{\circ} \mathrm{C}$ for several hours. It was fluorinated following the procedure described in Ref. [10] First it was transferred into a Monel reactor and heated to $260^{\circ} \mathrm{C}$ in flowing Argon. The gas was then switched to a mixture of fluorine gas (20\%) and nitrogen $(80 \%)$ for 10 hours at constant total flow rate of $30 \mathrm{sccm}$.

PEO (consists of repeating $-\mathrm{O}-\mathrm{CH}_{2}-\mathrm{CH}_{2}$ units) powder with a molecular weight of $30,000 \mathrm{~g} / \mathrm{mol}$ was purchased from Aldrich and used without further processing. PEO/SWNT composite membranes with F-SWNT loading of 1, 4, 6, and 10wt $\%$ and control samples (pure PEO) were prepared under the same conditions by roll-cast [16]. The roll-cast system comprises two opposing parallel rollers made of Teflon and stainless steel, respectively, and an electrical motor. The gap distance between the Teflon and the passive stainless steel roller which is placed on a translational stage can be adjusted using a micrometer to obtaine the desired film thickness. FSWNT was first suspended in isopropanol by sonication and was then mixed with a clear $\mathrm{PEO} /$ methanol solution. They were dropped slowly onto the Teflon roller using a pipette while it's rotating. A solid film formed after evaporation of the solvent. The film was peeled off, folded and 
cut to $0.2 \times 1 \times 7 \mathrm{~mm}$ samples for mechanical test. The samples are considerably more uniform than those formed using pristine CNTs.

To examine the dispersion of the nanotubes, samples were microtomed into membranes $\sim 90 \mathrm{~nm}$ in thickness and were studied by TEM. Within the limited view of high resolution TEM, no conglomeration of the F-SWNTs was observed. The nanotube bundles did not show any preferred orientation in the polymer matrix. Differential scanning calorimetry (DSC) measurements were performed using a Perkin-Elmer Pyris 1 DSC system. The results obtained in the first heating cycle are shown in Figure 2. For the control sample, an endothermic peak centered at $72.0^{\circ} \mathrm{C}$ was observed upon heating. This is attributed to melting of the crystalline phase of the semi-crystalline PEO. The measured latent heat which reflects the amount of crystalline phase in the sample is $135.3 \mathrm{~J} / \mathrm{g}$ (normalized by the total weight of the semi-crystalline sample). Both the melting temperature and the latent heat are consistent with the values reported in the literature. [17] For composites with F-SWNT loading $\leq 6 \mathrm{wt} \%$, a single endothermic peak was observed with a slightly lower melting temperature of $66-65^{\circ} \mathrm{C}$. The sample with $10 \mathrm{wt} \%$ of F-SWNT showed two endothermic peaks centered at $72.3^{\circ} \mathrm{C}$, the melting temperature of pure crystalline PEO phase, and $65^{\circ} \mathrm{C}$, respectively. These results indicate that single-phase F-SWNT/PEO blend was formed at $\leq 6 \mathrm{wt} \%$ F-SWNT loading, while phase separation (into regions of pure PEO and regions of PEO/FSWNT) occurred at higher F-SWNT loading due to conglomeration of F-SWNTs. No significant change in the crystallinity of the PEO matrix was observed. The measured latent heats are $135.3 \mathrm{~J} / \mathrm{g}$, 118.7J.g and 146.6J/g for the control sample (pure PEO), and PEO blended with 1 and $4 \mathrm{wt} \%$ of FSWNTs, respectively.

The mechanical properties were investigated using a dynamic mechanical analyzer (PerkinElmer DMA7e) system using either the oscillatory mode $(1 \mathrm{~Hz})$ or the tensile stress-strain mode in 
the temperature range of $-150^{\circ} \mathrm{C}$ to $60^{\circ} \mathrm{C}$. For dynamic measurement, an oscillating sinusoidal force with a frequency of $1 \mathrm{~Hz}$ and amplitude of $60 \mathrm{mN}$ was applied to the sample. By analysis of the corresponding strain, the storage modulus (E') and loss modulus (E') were obtained. In the tensile test, the load was increased at a rate of $100 \mathrm{mN} / \mathrm{min}$. For each F-SWNT concentration, several samples were measured under the same conditions. Figure 3 shows the storage modules of one set of samples obtained in the first cooling and heating cycle. The room temperature E's from all the samples measured are plotted in Figure 3 inset as E' versus wt $\%$ of F-SWNT loading. The storage moduli of the composites are significantly higher than that of the pure PEO in the entire temperature range of the experiment, and increased monotonically with increasing F-SWNT loading. As shown in Figure 3 inset, the averaged 300K E' (over several samples with the same FSWNT concentration) increased from $0.3 \mathrm{GPa}$ for pure $\mathrm{PEO}$ to $1.2 \mathrm{GPa}$ for PEO blended with $4 \mathrm{wt} \%$ of F-SWNT. In contrast, when pristine carbon nanotubes were used without fluorination, no systematic enhancement of the mechanical properties was observed at the same nanotube loading level.

Figure 4 shows the tensile stress-strain curves of a composite with $1 \mathrm{wt} \%$ of F-SWNT and a control sample, measured at a rate of $100 \mathrm{mN} / \mathrm{min}$. The sample with $1 \mathrm{wt} \%$ of F-SWNT has a significantly higher $(\sim 3 \mathrm{x})$ yield strength compared to that of the control sample. The elastic modulus, obtained from fitting the initial slope of the stress-strain curve, is $5.95 \times 10^{7} \mathrm{~Pa}$ for the control sample and $1.47 \times 10^{8} \mathrm{~Pa}$ for the PEO reinforced with $1 \mathrm{wt} \%$ of F-SWNTs. The mechanical properties of the composites were measured in two orthogonal directions (parallel and perpendicular to the direction of the roller motion) were measured using samples from the same batch. No noticeable anisotropy was observed. 
The above results show that adding a small amount of F-SWNTs drastically enhances the mechanical properties of the polymer matrix. These are in contrast to the previous studies of composites formed using pristine nanotubes where the change in the elastic properties are, in general, insignificant even at very high nanotube loading. Two recent studies reported moderate increase in the storage modulus in polymer composites blended with multi-wall carbon nanotubes (MWNTs). The room temperature E' was found to increase by $\sim 60 \%$ (from $\sim 6 \mathrm{GPa}$ to $10 \mathrm{GPa}$ ) when $50 \mathrm{wt} \%$ of MWNTs was added to poly(vinyl alcohol) (PVOH) matrix by solution cast. [20] A separate study reported a factor of $\sim 2$ increase in the storage modulus when $26 \mathrm{wt} \%$ of MWNTs was added to poly(methyl methacrylate) (PMMA) [21] (both studies reported larger changes of E' above room temperature).

The large increase of the elastic modulus at low nanotube loading suggests effective load transfer from the matrix to the F-SWNTs in the current system. The results were analyzed using the equation developed for short-fiber composites [22, 23]:

$$
\mathrm{E}=\mathrm{C}_{l} \mathrm{C}_{\theta} \mathrm{V}_{\mathrm{f}} \mathrm{E}_{\mathrm{f}}+\left(1-\mathrm{V}_{\mathrm{f}}\right) \mathrm{E}_{\mathrm{m}}
$$

where $\mathrm{E}, \mathrm{E}_{\mathrm{f}}, \mathrm{E}_{\mathrm{m}}$ are the moduli of the composite, fiber, and polymer matrix, and $\mathrm{C}_{l}$ and $\mathrm{C}_{\theta}$ are coefficients related to the fiber length and orientation, respectively. For three-dimensionally randomly oriented fibers, as in the case of the F-SWNTs in PEO, $\mathrm{C}_{\theta}$ is about 0.2 [23, 24]. Using the average elastic modulus of $0.3 \mathrm{GPa}$ measured for the control sample at room temperature as the value for $\mathrm{E}_{\mathrm{m}}$, and assuming unity for $\mathrm{C}_{l}$, the elastic modulus of the F-SWNT bundle is calculated to be $\sim 0.1 \mathrm{TPa}$. The value is higher when a lower number is used for $\mathrm{C}_{l}$. This is surprisingly close to the reported value of $1 \mathrm{TPa}$ calculated [25] and the upper value derived experimentally [3] for an isolated CNT, especially when considering that defects were not taking into accounts in the calculation. In addition, SWNT bundles with weak van der Waals inter-tube bonding were used in 
the current experiments, rather than isolated SWNTs. The close agreement between elastic modulus calculated from the equation derived for short-fiber composites and the value measured from an isolated nanotube and nanotube bundles [26] indicates that there is an efficient load transfer between the F-SWNTs and PEO matrix. In comparison, in the PVOH/CNT system, the calculated elastic modulus of the CNTs is only $150 \mathrm{MPa}$ [20]. The lower value was attributed to the weak interfacial coupling. In addition the results reported here show that side-wall fluorination does not significantly reduce the mechanical performances of the carbon nanotubes. At this point, it is not clear whether the enhanced load transfer, compared to composites using pristine CNTs, is due to chemical bonding between the fluorine and PEO or/and due to improved dispersion of the FSWNTs in the polymer matrix and consequently increased entanglement between nanotubes and the polymeric chains.

In summary, we show that composites with improved uniformity and dispersion can be

formed using chemically functionalized carbon nanotubes. A significant enhancement of the mechanical properties was obtained at low nanotube loading. In contrary to previous results from pristine nanotubes, the composites show efficient load transfer between the fillers and matrix.

\section{ACKNOWLEDGEMENT:}

This work was supported by a grant from NASA (NAG-1-01061) and the Office of Naval Research under a MURI grant at UNC (N00014-98-1-0597). H.Z. Geng was supported by a Merit Scholarship from UNC. We thank E. Samulski for suggesting the roll-cast method and E.L. Thomas for helpful discussions. 


\section{REFERNCES:}

1. S. Iijima, Nature, 1991. 354: p. 56-58.

2. M.S. Dresselhaus, G. Dresselhaus, and P. Avouris, eds. Carbon Nanotubes : Synthesis, Structure, Properties, and Applications. Topics in Applied Physics. Vol. 80. 2000, Springer-Verlag: Heidelberg.

3. E.W. Wong, P.E. Sheehan, and C.M. Lieber, Science, 1997 . 277(26): p. 1971-1975.

4. P.M. Ajayan and O. Zhou, Applications of Carbon Nanotubes, in Carbon Nanotubes : Synthesis, Structure, Properties, and Applications (Topics in Applied Physics, 80), M.S. Dresselhaus, G. Dresselhaus, and P. Avouris, Editors. 2000, Springer-Verlag: Heidelberg.

5. L.S. Schadler, S.C. Giannaris, and P.M. Ajayan, Appl. Phys. Lett., 1999. 73: p. 3842-3844.

6. C. Bower, R. Rosen, L. Jin, J. Han, and O. Zhou, Appl. Phys. Lett., 1999. 74(22): p. 33173319.

7. G.H. Haggenmueller R, Rinzler AG, Fischer JE, Winey KI, Chem. Phys. Lett, 2000. 330: p. 219-225.

8. L. Jin, C. Bower, and O. Zhou, Appl. Phys. Lett., 1998 . 73(9): p. 1197-1199.

9. O. Lourie, D.E. Cox, and H.D. Wagner, Phys. Rev. Lett., 1998. 81(8): p. 1638-1641.

10. E.T. Mickelson, C.B. Huffman, A.G. Rinzler, R.E. Smalley, R.H. Hauge, and J.L. Margrave, Chem. Phys. Lett, 1998. 296: p. 188-194.

11. M.A. Hamon, J. Chen, H. Hu, Y. Chen, M.E. Itkis, A.M. Rao, P.C. Eklund, and R.C. Haddon, Adv. Mat., 1999. 11: p. 834.

12. E.T. Mickelson, I.W. Chiang, J.L. Zimmerman, P.J. Boul, J. Lozano, J. Liu, R.E. Smalley, R.H. Hauge, and J.K. Margrave, J. Phy. Chem. B., 1999. 
13. A. Thess, R. Lee, P. Nikdaev, H. Dai, P. Petit, J. Robert, C. Xu, Y.H. Lee, S.G. Kim, A.G. Rinzler, D.T. Colbert, G.E. Scuseria, D. Tomanek, J.E. Fischer, and R.E. Smalley, Science, 1996 . 273: p. 483-487.

14. X.P. Tang, A. Kelinhammes, H. Shimoda, L. Fleming, C. Bower, S. Sinha, O. Zhou, and Y. Wu, Science, 2000. 228: p. 492.

15. O. Zhou, B. Gao, C. Bower, L. Fleming, and H. Shimoda, Mol. Crys. and Liq. Crys., 2000 . 340: p. 541-546.

16. R.J. Albalak and E.L. Thomas, Journal of Polymer Science: Part B: Polymer Physics, 1993. 31: p. 37-46.

17. M. Armand, J.Y. Sanchez, M. Gauthier, and Y. Choquette, in Electrochemistry of Novel Materials: Frontiers of Electrochemistry, J. Lipkowski and P.N. Ross, Editors. 1994, VCH.

18. E.P. Giannelis, in Biomimetic Materials Chemistry, S. Mann, Editor. 1996, VCH.

19. F. Croce, G.B. Appetecchi, L. Persi, and B. Scrosati, Nature, 1998. 394: p. 456.

20. M.S.P. Shaffer and A.H. Windle, Adv. Mat., 1999. 11(11): p. 937-941.

21. Z. Jin, K.P. Pramoda, G. Xu, and S.H. Goh, Chem. Phys, Lett, 2001. 337: p. 43-47.

22. H. Fukuda and K. Kawata, Fibre Sci. Tech., 1974. 7: p. 207-222.

23. T.W. Chou, Microstructural design of fiber composite. 1992, Cambridge: Cambridge University Press.

24. J. W.D. Callister, Materials science and enginnering: an introduction. third ed. 1994: Wiley.

25. J.P. Lu, Phys. Rev. Lett., 1997. 79(7): p. 1297-1300.

26. J.P. Salvetat, G. Andrew, D. Briggs, J.M. Bonard, R.R. Bacsa, A.J. Kulik, T. Stockli, N.A. Burnham, and L. Forro, Phy. Rev. Lett., 1999. 82(5): p. 944-947. 


\section{Figure Caption:}

Figure 1: Transmission electron microscope image of purified SWNT bundles before fluorination. The nanotubes were fabricated by the laser ablation method and were purified by reflux in peroxide solution followed by filtration. The bundles are $30-50 \mathrm{~nm}$ in diameter and are $\sim 10 \mu \mathrm{m}$ long.

Figure 2: Differential scanning calorimetry (DSC) data of pure PEO and PEO/F-SWNT composites. The endothermic peak on the heating curve is attributed to melting of the crystalline phase of the PEO matrix. The melting temperature is $72^{\circ} \mathrm{C}$ for the control sample with a latent heat is $135.3 \mathrm{~J} / \mathrm{g}$. The temperature is reduced to of $\sim 65^{\circ} \mathrm{C}$ for the composites. The re-appearance of a second endothermic peak at $\sim 72^{\circ} \mathrm{C}$ in the $10 \mathrm{wt} \% \mathrm{~F}-\mathrm{SWNT}$ composite indicates macroscopic phase separation into regions of pure PEO and regions of PEO/F-SWNTs.

Figure 3: The storage modules, E', of the F-SWNT composites measured using a dynamics mechanical analyzer at $1 \mathrm{~Hz}$. The inset shows the room temperature E' values versus wt\% of FSWNT loading from all the samples measured under the same conditions. The averaged $20^{\circ} \mathrm{C} \mathrm{E}$ (over several samples with the same F-SWNT concentration) increased from 0.3GPa for pure PEO to $1.2 \mathrm{GPa}$ for PEO blended with $4 \mathrm{wt} \%$ of F-SWNT.

Figure 4: The engineering tensile stress-strain curves measured at a rate of $100 \mathrm{~m} \mathrm{~N} / \mathrm{min}$. The sample with $1 w t \%$ of F-SWNT has $\sim 3 x$ higher yield strength compared to that of the pure PEO.

The elastic modulus is $5.95 \times 10^{7}$ and $1.47 \times 10^{8} \mathrm{~Pa}$ for the control and the PEO reinforced by $1 \mathrm{wt} \%$ of F-SWNTs, respectively. 


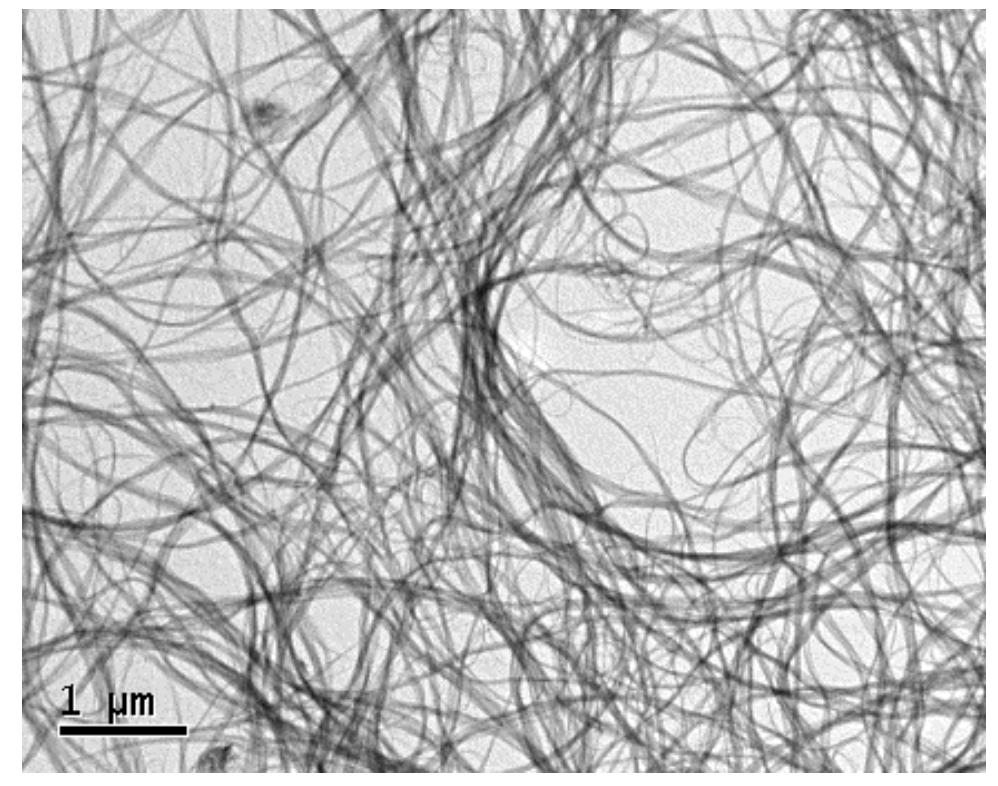




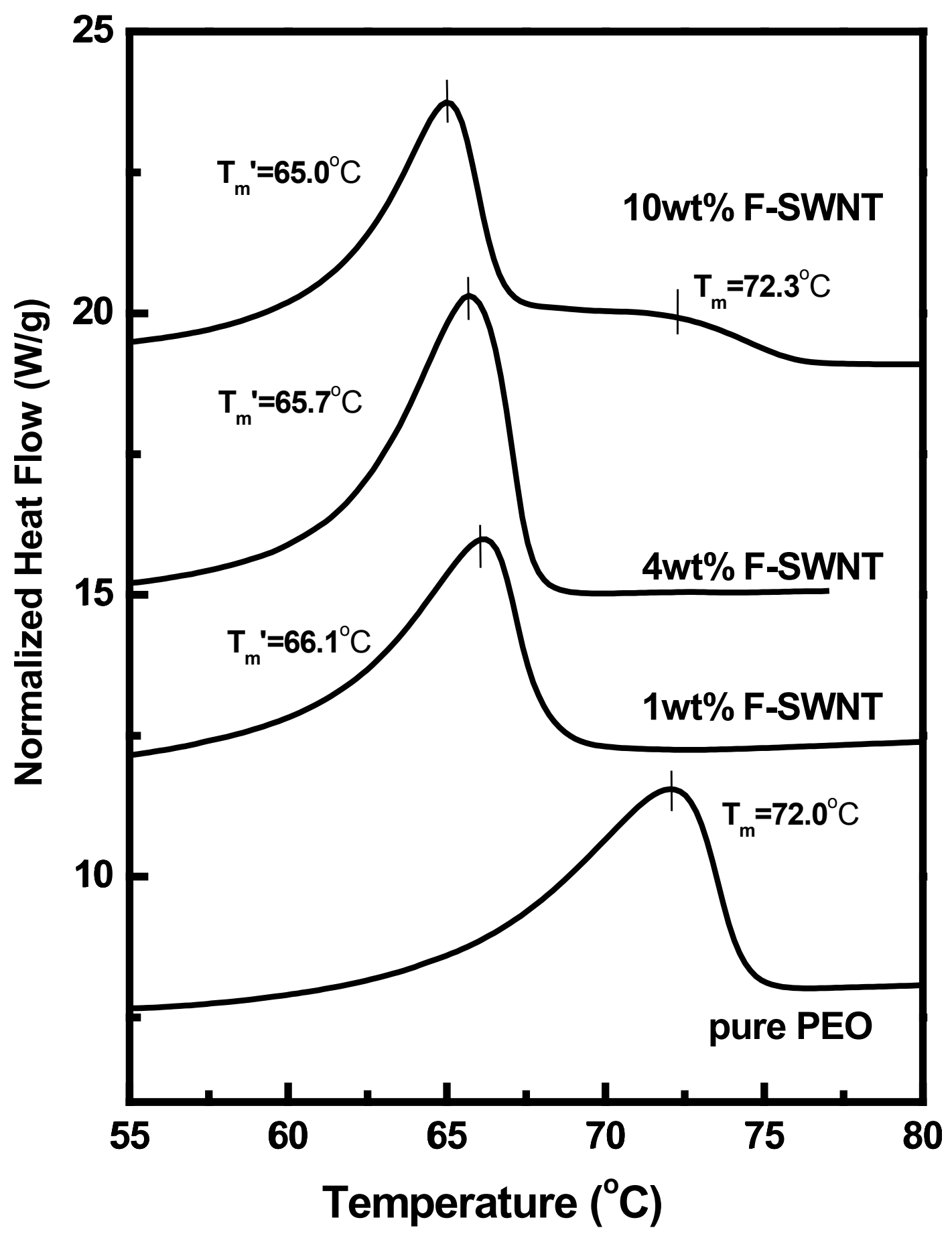




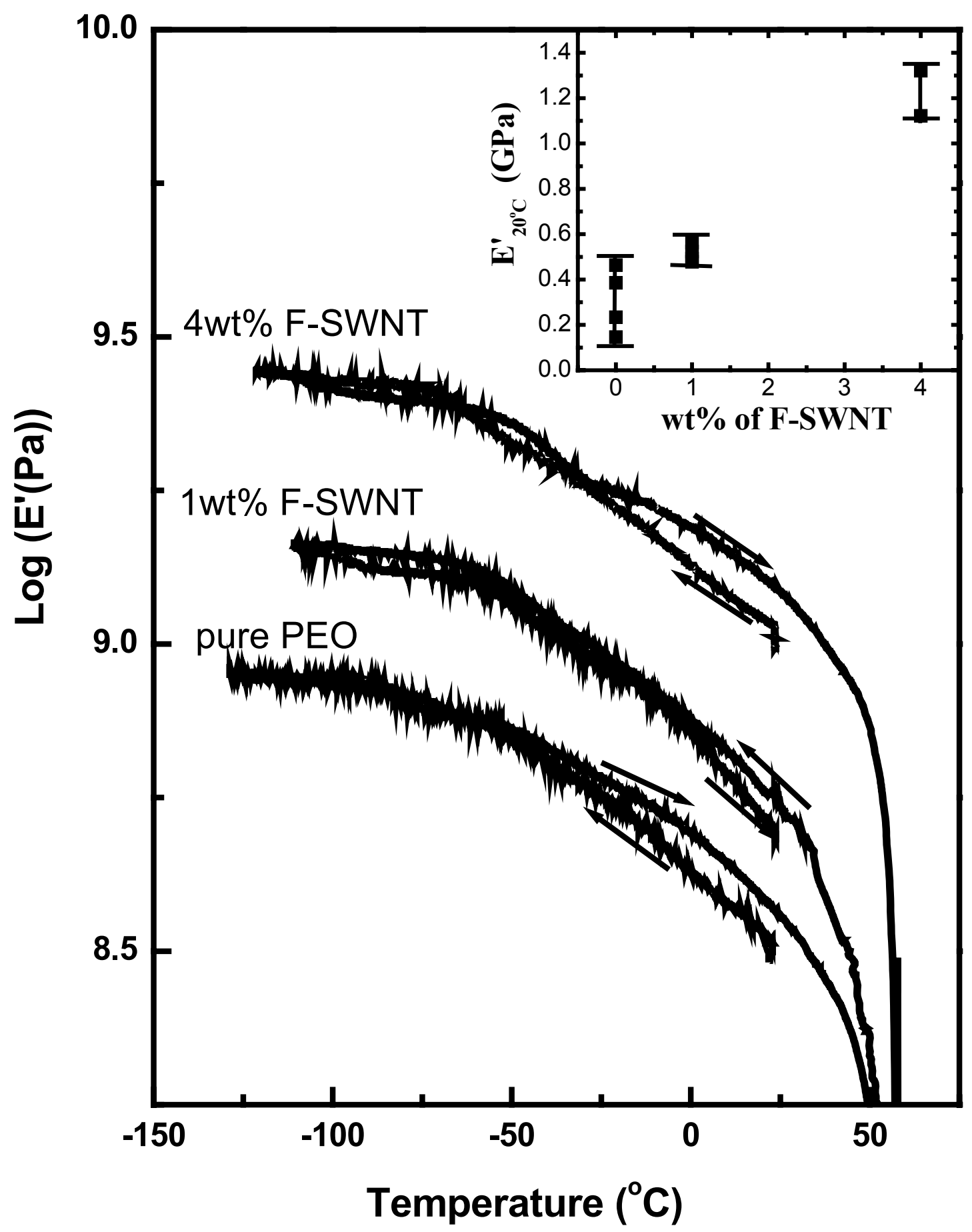




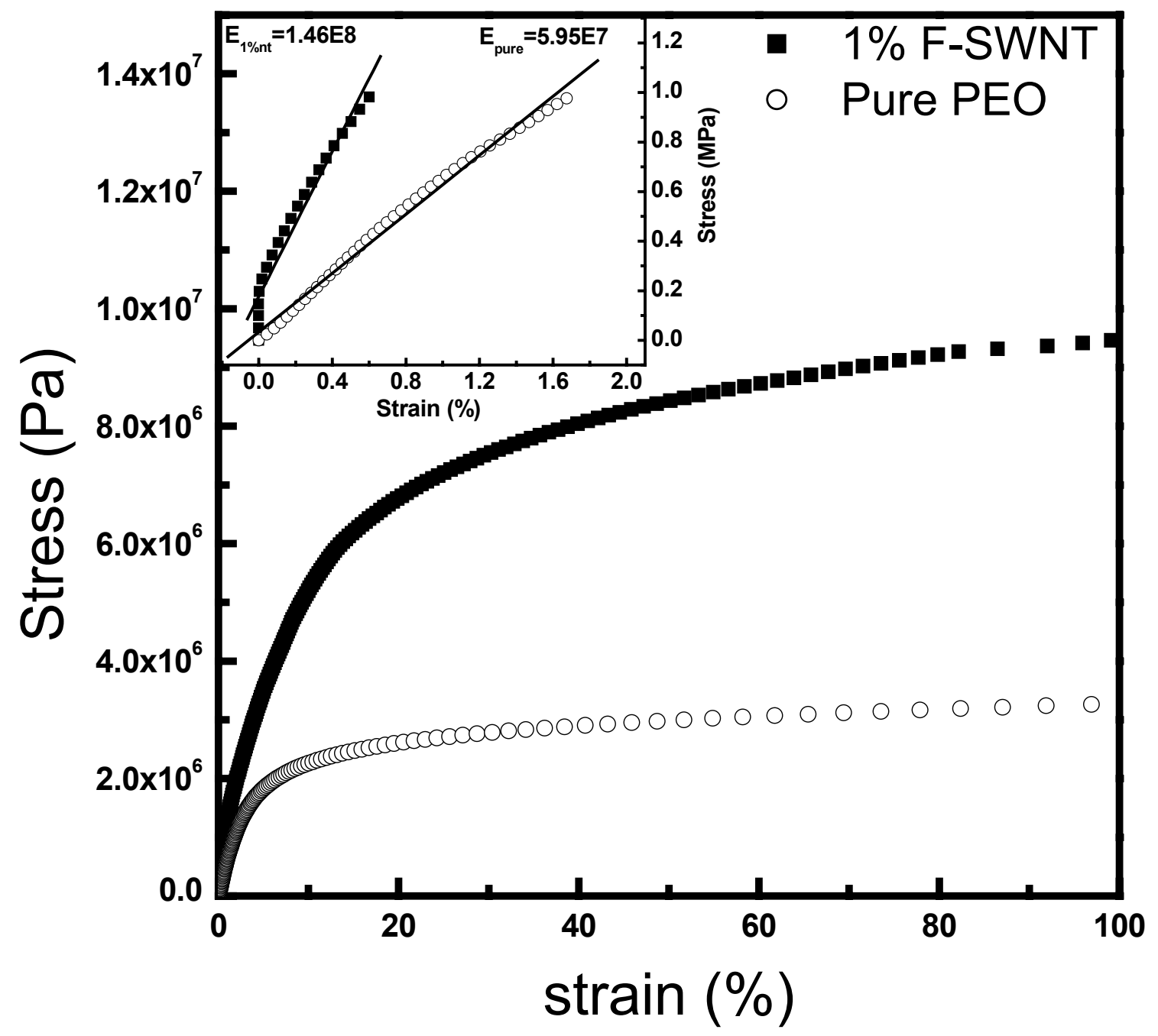

